\title{
Relay selection in cooperative networks with frequency selective fading
}

\author{
Qingxiong Deng ${ }^{*}$ and Andrew G Klein
}

\begin{abstract}
In this article, we consider the diversity-multiplexing tradeoff (DMT) of relay-assisted communication through correlated frequency selective fading channels. Recent results for relays in flat fading channels demonstrate a performance and implementation advantage in using relay selection as opposed to more complicated distributed space-time coding schemes. Motivated by these results, we explore the use of relay selection for the case when all channels have intersymbol interference. In particular, we focus on the performance of relaying strategies when multiple decode-and-forward relays share a single channel orthogonal to the source. We derive the DMT for several relaying strategies: best relay selection, random relay selection, and the case when all decoding relays participate. The best relay selection method selects the relay in the decoding set with the largest sum-squared relay-to-destination channel coefficients. This scheme can achieve the optimal DMT of the system under consideration and generally dominates the other two relaying strategies which do not always exploit the spatial diversity offered by the relays. Different from flat fading, we found special cases when the three relaying strategies have the same DMT. We further present a transceiver design which is proven to asymptotically achieve the optimal DMT. Monte Carlo simulations are presented to corroborate the theoretical analysis and to provide a detailed performance comparison of the three relaying strategies in channels encountered in practice.
\end{abstract}

Keywords: cooperative communication, relay selection, opportunistic relaying, diversity-multiplexing tradeoff, outage probability, frequency selective fading, intersymbol interference

\section{Introduction}

Cooperative relay networks have emerged as a powerful technique to combat multipath fading and increase energy efficiency [1,2]. To exploit spatial diversity in the absence of multiple antennas, several spatially separated singleantenna nodes can cooperate to form a virtual antenna array. Such systems usually employ half-duplex relays and come in two flavors [3-6]: those where the relays transmit on orthogonal channels so that transmission from the source and each relay is received separately at the destination, or those where a single non-orthogonal channel is shared between the source and relays so that all nodes may transmit on the same common channel at the same time. Here, we focus on the former class of systems which employ orthogonal relay channels, where the orthogonality is often accomplished through time division.

\footnotetext{
* Correspondence: qxdeng@wpi.edu

Department of Electrical and Computer Engineering, Worcester Polytechnic
} Institute, Worcester, MA 01609, USA



Cooperative relay systems with orthogonal channels typically either employ multiple orthogonal relay subchannels in conjunction with repetition coding, or all relays use a single orthogonal relay channel along with distributed space-time coding (DSTC) [7]. While the use of repetition codes is attractive for its simplicity, this approach requires relay scheduling and dedicated orthogonal channels for each relay which uses up precious system resources. On the other hand, when using a single orthogonal relay channel with DSTC, the scheduling of relays is of no concern, but DSTC requires synchronization between relays which is very difficult in distributed networks. Asynchronous forms of space-time coding have been proposed (e.g. [8]), but the decoding complexity may still be prohibitively complex to permit their use in low-cost wireless ad hoc networks. Furthermore, the non-linearity of most existing RF front-ends poses additional implementation challenges for DSTC-based approaches [9].

More recently, relay selection schemes have been proposed $[10,11]$ which use simple repetition coding, very 
Table 1 DMT of each selection scheme for $r L[0,1 / 2]$

\begin{tabular}{lcc}
\hline Selection & $(1-2 r)\left(L_{s d}+\sum_{i=1}^{K} \min \left\{L_{r_{i} d}, L_{s r_{i}}\right\}\right)$ & $d(r)$ when $\forall i, L_{r_{i} d}=L_{r d}, L_{s r_{i}}=L_{s r}$ \\
Best & $(1-2 r)\left(L_{s d}+\min \mathcal{D}\left\{\left(\min _{\mathbf{R}_{i} \in \mathcal{D}} L_{r_{i} d}\right)+\left(\sum_{\mathbf{R}_{i} \notin \mathcal{D}} L_{s r_{i}}\right)\right\}\right)$ & $(1-2 r)\left(L_{s d}+\min \left\{K L_{r d}, K L_{s s}\right\}\right)$ \\
Random & $(1-2 r)\left(L_{s d}+\min \mathcal{D}\left\{\left(\min _{\mathbf{R}_{i} \in \mathcal{D}} L_{r_{i} d}\right)+\left(\sum_{\mathbf{R}_{i} \notin \mathcal{D}} L_{s r_{i}}\right)\right\}\right)$ & $(1-2 r)\left(L_{s d}+\min \left\{L_{r d}, K L_{s r}\right\}\right)$ \\
All & & $(1-2 r)\left(L_{s d}+\min \left\{L_{r d r} K L_{s r}\right\}\right)$ \\
\hline
\end{tabular}

simple scheduling, and a single relay channel. Remarkably, these schemes can achieve the same diversity-multiplexing tradeoff (DMT) [12] as DSTC relaying, and can even outperform DSTC systems in terms of outage probability $[11,13]$. Using relay selection is an attractive alternative to avoid the spectral inefficiency of repetition coding and the increased decoding complexity required for DSTC.

Most existing cooperative diversity research assumes that the fading channels have flat frequency responses. In high data-rate wireless applications, however, the coherence bandwidth of the channels tends to be smaller than the bandwidth of the signal, resulting in frequency selective fading [14]. For such high rate communication in cooperative relay networks, existing techniques for flat fading channels need to be adapted, or new techniques need to be designed for frequency selective fading channels. In [15], the authors considered a system with a single amplify-and-forward (AF) relay over frequency selective channels, and proposed three DSTCs. In [16], the authors consider a multiple-AF-relay OFDM system and proposed a distributed space-frequency code. The three DSTCs in [15] and the distributed space-frequency code in [16] can achieve both cooperative diversity and frequency diversity where the frequency diversity through a relay is up to the minimum of the source-relay channel length and the relay-destination channel length. Simpler, non-DSTC approaches that employ relay selection have been proposed for communication through frequency-selective fading channels. For example, in $[17,18]$, uncoded OFDM is studied, and it was shown that if relay selection is done on a per-subcarrier basis, full spatial diversity can be achieved. However, neither of these OFDM-based relay selection methods were able to exploit the frequency diversity of the ISI channel [19]. A linearly precoded OFDM system was proposed in [20] which uses multiple amplify-forward relays with linear transmit precoding; a simulation-based study showed that two relay selection schemes exhibited a coding gain improvement compared to an orthogonal round-robbin relaying scheme.

This article investigates the performance limits of relay selection with frequency selective fading, and focuses on the DMT for single-carrier systems without transmit channel state information (CSI) and transmit precoding. We analyze three different relay selection methods, including best relay selection, random relay selection, and all-decoding-relay participation. The relays in these three methods use a single orthogonal subchannel with repetition coding. We derive the DMT for the relay selection methods and then propose a practical low-complexity system which asymptotically attains the DMT by using uncoded QAM with guard intervals between blocks along with linear zero-forcing (ZF) equalizers.

\section{System model}

\subsection{Channel model}

We consider a system as in Figure 1, which consists of a single source node $(\mathbf{S}), K$ relay nodes $\left(\mathbf{R}_{1}, 2, \ldots, K\right)$, and a single destination node (D). We assume that all nodes have the same average power constraint $P$ watts and transmission bandwidth $W \mathrm{~Hz}$. While this model has been wellstudied in the case of static flat channels [21], here the links between the nodes are assumed to be frequency selective quasi-static fading channels, modeled as complex FIR filters. In the subscript, we denote $s$ as source node, $r_{i}$ as $i$ th relay and $d$ as destination. Thus, the source-to-destination channel coefficients are contained in the vector $\boldsymbol{h}_{s d}$. Similarly, for $i \in 1,2, \ldots, K$, the source-to-relay $\mathbf{R}_{i}$ channels are contained in $\boldsymbol{h}_{s r_{i}}$ and the relay $\mathbf{R}_{i}$ to destination channels are contained in $\boldsymbol{h}_{r_{i} d}$. Most analyses of diversity through frequency selective channels focus on the case where the channel taps are i.i.d. [22,23]. Even when multiple paths in the continuous time channel experience independent fading, however, the channel taps themselves can be highly correlated due to pulse shaping [14]. In addition, pulse shaping typically causes the number of discrete time channel taps to be quite a bit larger than the number of (possibly independent) fading paths. To incorporate correlated fading-as well as the effects of path loss, shadowing and imperfect timing synchronization-we assume that the channel taps arise as $\boldsymbol{h}_{j k}=\boldsymbol{\Gamma}_{j k} \boldsymbol{\delta}_{j k}$ where $j k$ could be $s d$, $s r_{i}$ or $r_{i} d, \boldsymbol{\delta}_{j k} \sim \mathcal{C N}\left(\mathbf{0}, \mathbf{I}_{L j k}\right)$ represents the $L_{j k}$ independent fading paths. The autocovariance of the $M_{j k}$ channel taps can then be specified by appropriate choice of $\boldsymbol{\Gamma}_{j k} \in \mathbb{R}^{M_{j k} \times L_{j k}}$ whose maximum singular value and minimum singular value are denoted as $\xi_{j k, \max }$ and $\xi_{j k, \min }$, respectively. Without loss of generality, we assume that $\boldsymbol{\Gamma}_{j k}$ is full column rank and $M_{j k} \geq L_{j k}$ so that the number of coefficients in the effective channel impulse response may 


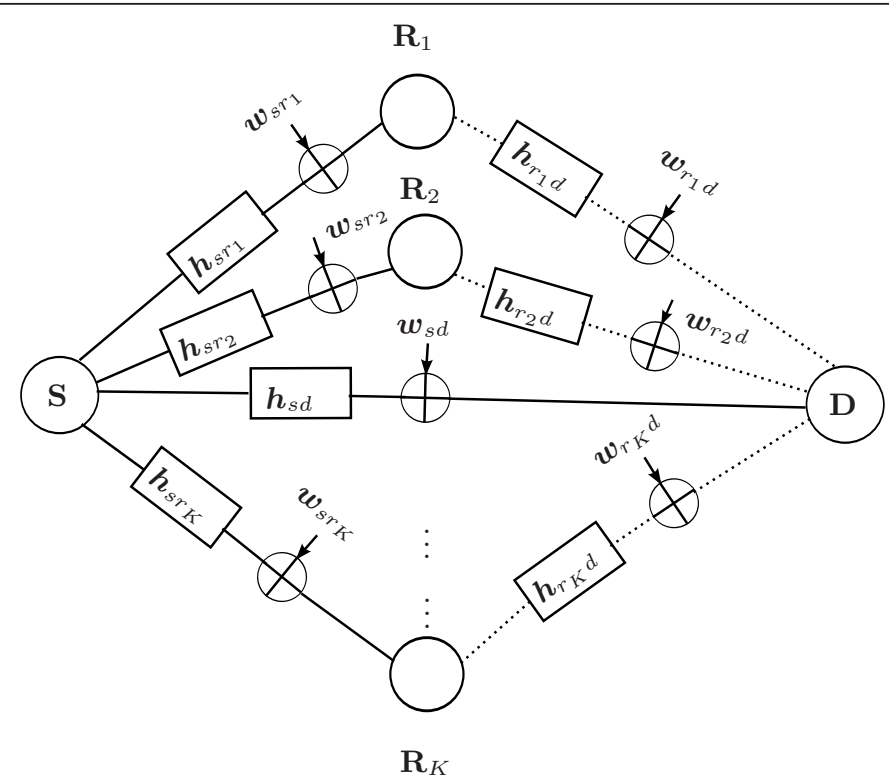

Figure 1 System model.

be greater than the number of fading paths in the physical channel.

The channel coefficients are assumed to be constant over a block and are independent from one block to the next. We assume perfect CSI at the destination and no CSI at the source. Furthermore, the transmission is presumed to be perfectly synchronized at the block level. In addition, all links have additive noise which is assumed to be mutually independent, zero-mean circularly symmetric complex Gaussian with variance $N_{0}$ and the discrete-time signal-to-noise ratio is defined as

$$
\rho \triangleq \frac{P}{W N_{\mathrm{o}}} .
$$

While the assumption of equal node powers and equal noise variances may seem impractical, the case of unequal powers and variances does not change the asymptotic high-SNR analysis which follows since these constants disappear in the derivation; consequently, we make this simplifying assumption to aid the clarity of the exposition.

\subsection{Diversity-multiplexing tradeoff}

The DMT has proven to be a useful theoretical tool that has considerably advanced the design of codes in the MIMO context. By restricting attention to system behavior in the high-SNR regime, DMT analysis permits a mathematically tractable comparison of various transmission and relaying schemes.

We define the outage probability as the probability that the mutual information $I$ between source and destination falls below rate $R$, and this is denoted as $\operatorname{Pr}[I<R$ $(\rho)]$. The multiplexing gain and the diversity gain are then defined as [14]

$$
r \triangleq \lim _{\rho \rightarrow \infty} \frac{R(\rho)}{\log \rho}, \quad d(r) \triangleq-\lim _{\rho \rightarrow \infty} \frac{\log (\operatorname{Pr}[I<R(\rho)])}{\log \rho},
$$

respectively, where $I$ is the mutual information between the source and the destination, and $R(\rho)$ denotes the source data rate which is assumed to scale as $R(\rho)=r \log \rho$. The notation $\doteq$ denotes asymptotic equality in the large $\rho$ limit with $A \doteq B$ meaning

$$
\lim _{\rho \rightarrow \infty} \frac{\log A}{\log \rho}=\lim _{\rho \rightarrow \infty} \frac{\log B}{\log \rho} .
$$

\subsection{Upper bound on the DMT}

The MFB assumes that the source only sends a single symbol $x[0]$ and the relay $\mathbf{R}_{i}$ only sends a single symbol $x_{r_{i}}[0]$ where $E\left[|x[0]|^{2}\right]=E\left[\left|x_{r_{i}}[0]\right|^{2}\right]=P / W$. For each source-to-relay link, the received signal at the relay is $\boldsymbol{y}_{r_{i}}=\boldsymbol{h}_{s_{i}} x[0]+\boldsymbol{w}_{r_{i}}$ where $\boldsymbol{w}_{r_{i}}$ is the noise at $\mathbf{R}_{i}$, and for each relay-to-destination link, the received signal at the destination can be expressed as $\boldsymbol{y}_{r_{i} d}=\boldsymbol{h}_{r_{i}} x_{r_{i}}[0]+\boldsymbol{w}_{d_{i}}$ where $\boldsymbol{w}_{d_{i}}$ is the noise at the destination $\mathbf{D}$ when $\mathbf{R}_{i}$ is transmitting. For the source-to-destination link, the received signal can be written as $\gamma_{s d}=\boldsymbol{h}_{s d} x[0]+\boldsymbol{w}_{d_{s}}$ where $\boldsymbol{w}_{d_{s}}$ is the noise at the destination $\mathbf{D}$ when the source $\mathbf{S}$ is transmitting. Thus, the mutual information between source and destination can be written as $I_{s d}=\log (1+\|$ $\boldsymbol{h}_{s d} \|^{2} \rho$ ). Similarly we can find the mutual information between source and $i$ th relay as $I_{s r_{i}}=\log \left(1+\left\|\boldsymbol{h}_{s r_{i}}\right\|^{2} \rho\right)$ 
and the mutual information between $i$ th relay and destination as $I_{r_{i} d}=\log \left(1+\left\|\boldsymbol{h}_{r_{i}}\right\|^{2} \rho\right)$. Define set $\mathcal{R}$ consisting of all $K$ relay nodes, and define a partition of $\mathcal{R}$ as $(\mathcal{V}, \mathcal{R} \backslash \mathcal{V})$. For the network as presented in the channel model with a single source $\mathbf{S}$ and a single sink $\mathbf{D}$, a cut $(\mathcal{S}, \mathcal{T})$ is defined as $\mathcal{S}=\{\mathbf{S}\} \cup \mathcal{V}$ and $\mathcal{T}=\{\mathbf{D}\} \cup(\mathcal{R} \backslash \mathcal{V})$. The capacity of a minimum cut of such a network can be upper bounded as [24]

$$
I_{\text {cut }}=\min _{\mathcal{V}}\left(I_{s d}+\sum_{\mathbf{R}_{i} \in(\mathcal{R} \backslash \mathcal{V})} I_{s r_{i}}+\sum_{\mathbf{R}_{i} \in(\mathcal{R} \backslash \mathcal{V})} I_{r_{i} d}\right) .
$$

The outage probability is lower bounded as

$$
\begin{aligned}
\mathbf{P}_{\text {out }} & \geq \operatorname{Pr}\left[I_{\text {cut }}<r \log \rho\right] \\
& =\operatorname{Pr}\left[\min _{\mathcal{V}}\left(I_{s d}+\sum_{\mathbf{R}_{i} \in(\mathcal{R} \backslash \mathcal{V})} I_{s_{i}}+\sum_{\mathbf{R}_{i} \in(\mathcal{R} \backslash \mathcal{V})} I_{r_{i} d}\right)<r \log \rho\right] \\
& =\max _{\mathcal{V}} \operatorname{Pr}\left[\left(I_{s d}+\sum_{\mathbf{R}_{i} \in(\mathcal{R} \backslash \mathcal{V})} I_{s_{i}}+\sum_{\mathbf{R}_{i} \in \mathcal{V}} I_{r_{i} d}\right)<r \log \rho\right] .
\end{aligned}
$$

For a particular partition of relay nodes $\mathcal{V}$, we have the outage probability

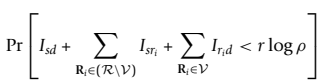

$$
\begin{aligned}
& =\operatorname{Pr}\left[\log \left(1+\left\|h_{s}\right\|^{2} \rho\right)+\sum_{\mathbf{R} \in \in(\mathcal{R} \mid V)} \log \left(1+\left\|h_{s i}\right\|^{2} \rho\right)+\sum_{R_{\in} \in \mathcal{V}} \log \left(1+\left\|h_{r i d}\right\|^{2} \rho\right)<r \log \rho\right] \\
& =\operatorname{Pr}\left[\log \left(\left(1+\left\|h_{s i l}\right\|^{2} \rho\right) \cdot \prod_{R_{R} \in(\mathbb{R} \mid V)}\left(1+\left\|h_{s}\right\|^{2} \rho\right) \cdot \prod_{R_{R} \in V}\left(1+\left\|h_{r i d}\right\|^{2} \rho\right)\right)<r \log \rho\right] \\
& \dot{\operatorname{Pr}}\left[\left\|\boldsymbol{h}_{s d}\right\|^{2} \rho+\sum_{\mathbf{R}_{i} \in(\mathcal{R} \backslash \mathcal{V})}\left\|\boldsymbol{h}_{s r_{i}}\right\|^{2} \rho+\sum_{\mathbf{R}_{i} \in \mathcal{V}}\left\|\boldsymbol{h}_{r_{i}}\right\|^{2} \rho<\rho^{r}\right] \\
& \doteq \operatorname{Pr}\left[\left\|\boldsymbol{\delta}_{s d}\right\|^{2} \rho+\sum_{\mathbf{R}_{4} \in(\mathcal{R} \backslash \mathcal{V})}\left\|\boldsymbol{\delta}_{s r_{i}}\right\|^{2} \rho+\sum_{\mathbf{R}_{i} \in \mathcal{V}}\left\|\boldsymbol{\delta}_{r_{i}}\right\|^{2} \rho<\rho^{r}\right](5) \\
& \doteq \rho^{-\left(L_{s d}+\sum_{\mathbf{R}_{i} \in(\mathcal{R} \backslash \mathcal{V})} L_{s r_{i}}+\sum_{\mathbf{R}_{i} \in \mathcal{V}} L_{r_{i} d}\right)(1-r)},
\end{aligned}
$$

where (3) follows from $\log A+\log B=\log (A B)$, (4) follows from the fact that $\operatorname{Pr}(a+b<c) \leq \operatorname{Pr}(a<c)$ for any a, $b, c \geq 0$, (5) follows from the fact that $\xi_{j k, \text { min }}^{2}\left\|\boldsymbol{\delta}_{j k}\right\|^{2} \leq\left\|\boldsymbol{h}_{j k}\right\|^{2} \leq \xi_{j k, \max }^{2}\left\|\boldsymbol{\delta}_{j k}\right\|^{2}$, and (6) holds as $\left\|\boldsymbol{\delta}_{s d}\right\|^{2}+\sum_{\mathbf{R}_{i} \in(\mathcal{R} \backslash \mathcal{V})}\left\|\boldsymbol{\delta}_{s r_{i}}\right\|^{2}+\sum_{\mathbf{R}_{i} \in \mathcal{V}}\left\|\boldsymbol{\delta}_{r_{i} d}\right\|^{2}$ is chi-square distributed with $L_{s d}+\sum_{\mathbf{R}_{i} \in(\mathcal{R} \backslash \mathcal{V})} L_{s r_{i}}+\sum_{\mathbf{R}_{i} \in \mathcal{V}} L_{r_{i} d}$ degrees of freedom.

Substituting (6) into (2), the outage probability is lower bounded as

$$
\mathrm{P}_{\text {out }} \doteq \rho^{-\min _{\mathcal{V}}\left(L_{s d}+\sum_{\mathbf{R}_{i} \in(\mathcal{R} \backslash \mathcal{V})} L_{s r_{i}}+\sum_{\mathbf{R}_{i} \in \mathcal{V}} L_{r_{i}} d\right)(1-r)} .
$$

Thus, the DMT is upper bounded as

$$
\begin{aligned}
d(r) & \leq \min _{\mathcal{V}}\left(L_{s d}+\sum_{\mathbf{R}_{i} \in(\mathcal{R} \backslash \mathcal{V})} L_{s r_{i}}+\sum_{\mathbf{R}_{i} \in \mathcal{V}} L_{r_{i} d}\right)(1-r) \\
& =\left(L_{s d}+\sum_{i=1}^{K} \min \left(L_{s r_{i}}, L_{r_{i} d}\right)\right)(1-r)
\end{aligned}
$$

as the minimum is attained when relay $\mathbf{R}_{i}$ is in $\mathcal{V}$ if $L_{r_{i} d}<L_{s r_{i}}$ and is not in $\mathcal{V}$ otherwise.

For half-duplex orthogonal relays, the multiplexing gain is halved [25] and the upper bound on the DMT for the same channel model but with half-duplex relaying becomes

$$
d(r) \leq\left(L_{s d}+\sum_{i=1}^{K} \min \left(L_{s r_{i}}, L_{r_{i} d}\right)\right)(1-2 r) .
$$

\section{Outage probability analysis of decode-and- forward relay system}

We now focus on the decode-and-forward relay system and derive its outage probability and DMT under several different relaying strategies. We assume the message sent by the source node is encoded to a block of $N$ source symbols. The relays operate in half-duplex mode, and thus do not transmit and receive at the same time. In addition, the relay nodes and the source use the same transmission bandwidth but employ time division so that the relays transmit on a channel orthogonal to the source. The transmission of a complete message is divided into two phases:

1. In phase one, the source broadcasts the message to the destination and the relays, and each relay attempts to decode the message.

2. In phase two, the source is silent. Depending on the relay selection strategy, some or all of the relays that successfully decoded the message (if any) forward the message to the destination.

The source and relays then alternate between these two phases; this is shown in Figure 2 for the case where two relays $\mathbf{R}_{1}$ and $\mathbf{R}_{K}$ participate in the second phase, and we note that the destination receives the composite signal corrupted by intersymbol interference, interblock interference, and additive noise. Only the relays which can correctly decode the message from the source can participate in forwarding the decoded message to the destination. We define such relays as decoding relays and they form a decoding set. In practice, the decision of whether the message is decoded successfully can be made with the help of a checksum (e.g. CRC) and we assume the 


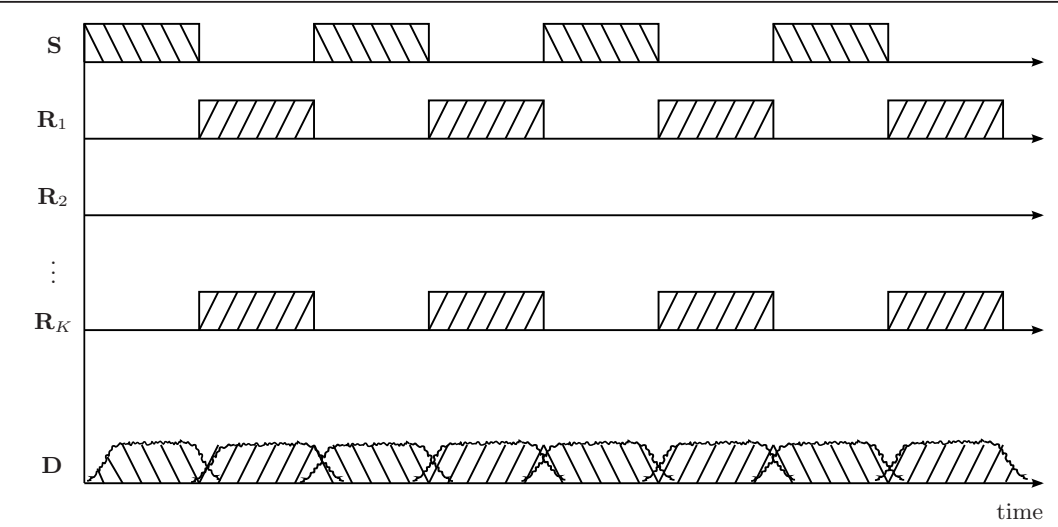

Figure 2 Transmission process

relays which pass this checksum do not contain any errors in the decoded message. We consider several relaying strategies in this article, including a best selection scheme, a random selection scheme, and a scheme where all decoding relays participate.

We continue to use the MFB to derive the upper bound on outage probability for the three relaying strategies and assume that a single symbol $x[0]$ is sent by the source.

In the first phase of transmission, the received signals at the destination and at each relay are given by

$$
\begin{aligned}
& y_{s d}=\boldsymbol{h}_{s d} x[0]+\boldsymbol{w}_{s d,} \\
& y_{r_{i}}=\boldsymbol{h}_{s r_{i}} x[0]+\boldsymbol{w}_{s r_{i}} .
\end{aligned}
$$

For classical direct transmission where the source transmits continuously without help from the relays, the mutual information between the source and $\mathbf{R}_{i}[14]$ would be $\log \left(1+\left\|\boldsymbol{h}_{s r_{i}}\right\|^{2} \rho\right)$ bits/s/Hz. In our system model, however, the use of time-division constrains the source to be silent half of the time which halves the mutual information but doubles the power, giving the mutual information between source and $\mathbf{R}_{i}$ in the first phase as

$$
I_{s r_{i}}=\frac{1}{2} \log \left(1+2\left\|\boldsymbol{h}_{s r_{i}}\right\|^{2} \rho\right) .
$$

Next, in phase two, each relay attempts to decode the message. Those relays which are able to successfully decode the message comprise the decoding set $\mathcal{D}$ where $\mathcal{D} \subseteq\left\{\mathbf{R}_{1}, \ldots, \mathbf{R}_{K}\right\}$. Depending on the relay selection strategy that is employed, some nodes in the decoding set will participate in the relaying.

To calculate the outage probability, we seek the overall mutual information $I$ between the source and the destination. Conditioning on the random set $D$, the total probability theorem gives the outage probability as

$$
\operatorname{Pr}[I<R]=\sum_{\mathcal{D}} \operatorname{Pr}[\mathcal{D}] \operatorname{Pr}[I<R \mid \mathcal{D}]
$$

with the summation over all possible decoding sets. To calculate the probability of a given decoding set $\mathrm{Pr}$ $[\mathcal{D}]$, first let

$$
b \triangleq \frac{2^{2 R}-1}{2 \rho}
$$

where we note that $b \doteq \rho^{2 r-1}$ and $0 \leq r \leq 1 / 2$. The probability that a relay node is in the decoding set is

$$
\begin{aligned}
\operatorname{Pr}\left[\mathbf{R}_{i} \in \mathcal{D}\right] & =\operatorname{Pr}\left[I_{s r_{i}}>R\right] \\
& =\operatorname{Pr}\left[\left\|\boldsymbol{h}_{s r_{i}}\right\|^{2}>b\right] \\
& \doteq \operatorname{Pr}\left[\left\|\boldsymbol{\delta}_{s r_{i}}\right\|^{2}>b\right] \\
& \doteq e^{-b} \sum_{k=0}^{L_{s r_{i}}-1} \frac{b^{k}}{k !}
\end{aligned}
$$

where the penultimate asymptotic equality follows from $\xi_{s r_{i}, \min }^{2}\left\|\boldsymbol{\delta}_{s r_{i}}\right\|^{2} \leq\left\|\boldsymbol{h}_{s r_{i}}\right\|^{2} \leq \xi_{s r_{i}, \max }^{2}\left\|\boldsymbol{\delta}_{s r_{i}}\right\|^{2}$ and the last asymptotic equality follows as $\left\|\boldsymbol{\delta}_{s r_{i}}\right\|^{2}$ is chi-square distributed with $L_{s r_{i}}$ degrees of freedom. Since each relay independently decodes the message, and since the channels from source to each relay are independent, the probability of the decoding set is

$$
\begin{aligned}
\operatorname{Pr}[\mathcal{D}] & \doteq \prod_{\mathbf{R}_{i} \notin \mathcal{D}}\left(1-e^{-b} \sum_{k=0}^{L_{s_{r_{i}}}-1} \frac{b^{k}}{k !}\right) \prod_{\mathbf{R}_{i} \in \mathcal{D}} e^{-b} \sum_{k=0}^{L_{s_{r_{i}}}-1} \frac{b^{k}}{k !} \\
& =b^{\Sigma_{\mathbf{R}_{i}} \notin \mathcal{D} L_{s_{r_{i}}}} .
\end{aligned}
$$

Referring back to (12), we now need to calculate $\operatorname{Pr}[I<R \mid \mathcal{D}]$, which depends on the particular choice of relay selection strategy. Next, we complete the outage probability and DMT derivation for each of the three selection strategies. 


\subsection{Best relay selection DMT}

We first analyze the outage of the best relay selection scheme, where the "best" relay is defined as the one with the largest sum-squared relay-to-destination channel coefficients. The chosen relay uses repetition coding, and simply forwards the decoded signal to the destination in phase two. The best relay selection process can be completed either centrally at the destination or in a distributed fashion by relays, as follows:

- Centralized selection: In turn, each decoding relay transmits some known information to the destination, and the destination estimates each relay-to-destination channel. The destination chooses the relay with the largest sum-squared relay-to-destination channel coefficients, and feeds back this decision to the relays. The feedback requires $|\mathcal{D}|$ bits and is assumed to be fed back reliably.

- Distributed selection: The relay-to-destination channel and the destination-to-relay channel are assumed to be the same due to reciprocity. The destination broadcasts some known information to all the relays, each of which individually estimate its relay-to-destination channel. Each relay waits for a time duration which is inversely proportional to its sum-squared relay-destination channel coefficients before sending its signal to the destination, so the relay with the largest sum-squared relay-to-destination channel will be the first to send its signal to the destination. Other relays do not start transmission if they overhear any signal from the best relay. The detailed process for this distributed relay selection is discussed in [10].

The system designer may choose which of these two approaches to adopt depending on the application. The centralized selection might consume more time since the channels between relays and destination would need to be estimated sequentially. Centralized selection also puts more estimation load on the destination. Distributed selection, on the other hand, is more spectrally efficiently since relays concurrently estimate the channels; however, the relays need to resolve collisions which may complicate the implementation. The practical details of the selection process itself-such as the overhead in performing the selection, as well as the possibility of poor channel estimates that result in a sub-optimal relay selection-are beyond the scope of the present study. Throughout our analysis, we assume that the best relay is always selected with negligible overhead.

Again, transmission takes place in two alternating phases, where the received signals in the first phase are given by (10) and (11). Here, however, only the selected relay participates in the second phase. Let the selected relay index be $m$ and denote its relay-destination channel coefficients as $\boldsymbol{h}_{r_{m} d}$ so that

$$
\left\|\boldsymbol{h}_{r_{m} d}\right\|^{2} \triangleq \max _{\mathbf{R}_{i} \in \mathcal{D}}\left\|\boldsymbol{h}_{r_{i} d}\right\|^{2} .
$$

The received signal at the destination becomes

$$
\boldsymbol{y}_{r d}=\boldsymbol{h}_{r_{m} d} x[0]+\boldsymbol{w}_{r d} .
$$

Due to the use of repetition coding by the selected relay and the orthogonality of the source-destination and source-relay channels, the conditional mutual information of the best relay selection scheme can be written as

$$
I_{\text {best }}=\frac{1}{2} \log \left(1+2 \rho\left(\max _{\mathbf{R}_{i} \in \mathcal{D}}\left\|\boldsymbol{h}_{r_{i} d}\right\|^{2}+\left\|\boldsymbol{h}_{\text {sd }}\right\|^{2}\right)\right) .
$$

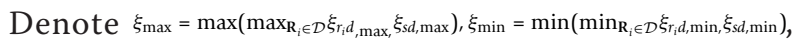
we have the following upper bound and lower bound on $I_{\text {best }}$ as

$$
\begin{aligned}
& \frac{1}{2} \log \left(1+2 \rho \xi_{\min }^{2}\left(\max _{\mathbf{R}_{i} \in \mathcal{D}}\left\|\boldsymbol{\delta}_{r_{i} d}\right\|^{2}+\left\|\boldsymbol{\delta}_{s d}\right\|^{2}\right)\right) \leq I_{\text {best }} \\
& \leq \frac{1}{2} \log \left(1+2 \rho \xi_{\max }^{2}\left(\max _{\mathbf{R}_{i} \in \mathcal{D}}\left\|\boldsymbol{\delta}_{r_{i} d}\right\|^{2}+\left\|\boldsymbol{\delta}_{s d}\right\|^{2}\right)\right) .
\end{aligned}
$$

Let $Y \triangleq\left\|\boldsymbol{\delta}_{s d}\right\|^{2}$ and $f_{Y}(y)$ be the pdf of $Y$ which is chisquare distributed with $L_{s d}$ degrees of freedom. The conditional outage probability for best relay selection is then

$$
\begin{aligned}
& \operatorname{Pr}\left[I_{\text {best }}<R \mid \mathcal{D}\right]=\operatorname{Pr}\left[\left(\max _{\mathbf{R}_{i} \in \mathcal{D}}\left\|\boldsymbol{h}_{r_{i} d}\right\|^{2}+\left\|\boldsymbol{h}_{s d}\right\|^{2}\right)<b\right] \\
& \doteq \operatorname{Pr}\left[\left(\max _{\mathbf{R}_{i} \in \mathcal{D}}\left\|\boldsymbol{\delta}_{r_{i} d}\right\|^{2}+Y\right)<b\right] \\
& =\int_{0}^{b} \operatorname{Pr}\left[\max _{\mathbf{R}_{i} \in \mathcal{D}}\left\|\boldsymbol{\delta}_{r_{i} d}\right\|^{2}<b-\gamma\right] f_{Y}(\gamma) d y \\
& =\int_{0}^{b}\left(\prod_{\mathbf{R}_{i} \in \mathcal{D}} \operatorname{Pr}\left[\left\|\boldsymbol{\delta}_{r_{i}}\right\|^{2}<b-\gamma\right]\right) \frac{1}{\left(L_{s d}-1\right) !} y^{L_{s d}-1} e^{-\gamma} d y \\
& =\int_{0}^{b}\left[\prod_{\mathbf{R}_{i} \in \mathcal{D}} e^{-(b-\gamma)}\left(\sum_{k=L_{r_{i}}}^{+\infty} \frac{(b-\gamma)^{k}}{k !}\right)\right] \frac{1}{\left(L_{s d}-1\right) !} y^{L_{s d}-1} e^{-y} d y \\
& =\int_{0}^{1} e^{-b}\left[\prod_{\mathbf{R}_{i} \in \mathcal{D}}\left(\sum_{k=L_{r_{i}}}^{+\infty} b^{k} \frac{(1-\alpha)^{k}}{k !}\right)\right] \frac{1}{\left(L_{s d}-1\right) !}(b \alpha)^{L_{s, d}-1} b d \alpha \\
& \doteq b^{L_{s d}+\sum_{\mathbf{R}_{i} \in \mathcal{D}} L_{r_{i} d}} \int_{0}^{1} \frac{(1-\alpha)^{\sum_{\mathbf{R}_{i} \in \mathcal{D}} L_{r_{i} d}} \alpha^{L_{s d-1}}}{\left(\prod_{\mathbf{R}_{i} \in \mathcal{D}} L_{r_{i} d} !\right)\left(L_{s d}-1\right) !} d \alpha
\end{aligned}
$$




$$
\doteq b^{L_{s d}+\sum_{\mathrm{R}_{i} \in \mathcal{D}} L_{r_{i} d}}
$$

where (18) follows by applying (16), (19) follows from [26, equation 2.321], (20) follows from the change of variable $y=\alpha b$ with $0 \leq \alpha \leq 1$, (21) comes by dropping terms in the polynomial of $b$ with order higher than $L_{r_{i}} d$, and (22) follows from the fact that the integration in (21) is not a function of $b$.

Substituting (13) and (22) into (12), the outage of best relay selection is then

$$
\begin{aligned}
& \operatorname{Pr}\left[I_{\text {best }}<R\right]=\sum_{\mathcal{D}} \operatorname{Pr}\left[I_{\text {best }}<R \mid \mathcal{D}\right] \operatorname{Pr}[\mathcal{D}] \\
& \doteq \sum_{\mathcal{D}} b^{L_{s d}+\sum_{\mathrm{R}_{i} \in \mathcal{D}} L_{r_{i} d}+\sum_{\mathrm{R}_{i} \notin \mathcal{D}} L_{s r_{i}}} \\
& \doteq b^{L_{s d}+\min _{\mathcal{D}}\left\{\left(\sum_{\mathrm{R}_{i} \in \mathcal{D}} L_{r_{i} d}\right)+\left(\sum_{\mathbf{R}_{i} \notin \mathcal{D}} L_{s r_{i}}\right)\right\}} \\
& \doteq \rho^{(2 r-1)\left(L_{s d}+\min _{\mathcal{D}}\left\{\left(\sum_{\mathrm{R}_{i} \in \mathcal{D}} L_{r_{i} d}\right)+\left(\sum_{\mathbf{R}_{i} \notin \mathcal{D}} L_{s r_{i}}\right)\right\}\right)} \\
& \doteq \rho^{(2 r-1)\left(L_{s d}+\sum_{i-1}^{K} \min \left(L_{r_{i} d}, L_{s r_{i}}\right)\right),}
\end{aligned}
$$

where (24) follows as the minimum in (23) is attained when relay $\mathbf{R}_{i}$ is in decoding set if $L_{r_{i} d}<L_{s r_{i}}$ and is not in decoding set otherwise. We see that full spatial diversity is achieved by this relay selection method since there are $K+1$ terms in (24), but the achieved frequency diversity through each relay is the minimum of the length of the source-to-relay and relay-to-destination channels.

\subsection{Random relay selection DMT}

In this subsection, we analyze the outage of a random relay selection scheme, where a random relay in the decoding set handles the forwarding. While this strategy would appear to be suboptimal compared to the best relay selection scheme, random selection is attractive for its simplicity and the fact that it requires no feedback nor CSI. In random selection, the probability of a decoding relay being selected as the forwarding relay is $1 /|\mathcal{D}|$. The chosen relay employs repetition coding for the second phase of transmission. Similar to Section 3.1, this relay selection method can also be operated in a centralized mode or a distributed mode. Under centralized mode, there is no need to estimate the relay-to-destination channel, and the destination broadcasts the index number of a randomly selected relay in the decoding set; in distributed mode, each decoding relay waits for a random time which is uniformly distributed within a range with the maximum predefined by the system, and the first to transmit becomes the chosen relay. The mutual information conditioned on selecting relay $\mathbf{R}_{i} \in \mathcal{D}$ can be written as

$$
I_{\text {random }}=\frac{1}{2} \log \left(1+2 \rho\left(\left\|\boldsymbol{h}_{r_{i} d}\right\|^{2}+\left\|\boldsymbol{h}_{s d}\right\|^{2}\right)\right) .
$$

We have

$$
\begin{aligned}
& \frac{1}{2} \log \left(1+2 \rho \min \left\{\xi_{r_{i} d, \min ,}^{2} \xi_{s d, \min }^{2}\right\}\left(\left\|\boldsymbol{\delta}_{r_{i} d}\right\|^{2}+\left\|\boldsymbol{\delta}_{s d}\right\|^{2}\right)\right) \leq I_{\text {random }} \\
& \leq \frac{1}{2} \log \left(1+2 \rho \max \left\{\xi_{r_{i} d, \min ,}^{2} \xi_{s d, \min }^{2}\right\}\left(\left\|\boldsymbol{\delta}_{r_{i} d}\right\|^{2}+\left\|\boldsymbol{\delta}_{s d}\right\|^{2}\right)\right) .
\end{aligned}
$$

Let $Y \triangleq\left\|\boldsymbol{\delta}_{s d}\right\|^{2}$ and $f_{Y}(y)$ be the pdf of $Y$ which is chisquare distributed with $L_{s d}$ degrees of freedom. The conditional outage probability for the random relay selection method is

$$
\begin{aligned}
& \operatorname{Pr}\left[I_{\text {random }}<R \mid \mathcal{D}\right]=\sum_{\mathbf{R}_{i} \in \mathcal{D}} \frac{1}{|\mathcal{D}|} \operatorname{Pr}\left[I_{\text {random }}<R \mid \mathbf{R}_{i}, \mathcal{D}\right] \\
&=\sum_{\mathbf{R}_{i} \in \mathcal{D}} \frac{1}{|\mathcal{D}|} \operatorname{Pr}\left[\left\|\boldsymbol{h}_{r_{i} d}\right\|^{2}+\left\|\boldsymbol{h}_{s d}\right\|^{2}<b\right](27) \\
& \doteq \sum_{\mathbf{R}_{i} \in \mathcal{D}} \frac{1}{|\mathcal{D}|} b^{L_{s d}+L_{r_{i} d}} \\
& \doteq b^{L_{s d}+\min \mathbf{R}_{i} \in \mathcal{D} L_{r_{i} d}},
\end{aligned}
$$

where (27) follows from the same steps used in going from (17) to (22), but with only one relay in the decoding set. From (28), we see that within the decoding set, random relaying offers no spatial diversity but only frequency diversity, where the diversity order equals the shortest channel length. Substituting (13) and (28) into (12), the outage of the random relay selection is

$$
\begin{aligned}
& \operatorname{Pr}\left[I_{\text {random }}<R\right]=\sum_{\mathcal{D}} \operatorname{Pr}\left[I_{\text {random }}<R \mid \mathcal{D}\right] \operatorname{Pr}[\mathcal{D}] \\
& \doteq \sum_{\mathcal{D}} b^{L_{s d}+\min _{R_{i} \in \mathcal{D}} L_{r_{i} d}+\sum_{R_{i} \notin \mathcal{D}} L_{s r_{i}}} \\
& \doteq \rho^{(2 r-1)\left(L_{s d}+\min _{\mathcal{D}}\left\{\left(\min _{R_{i} \in \mathcal{D}} L_{r_{i} d} d\right)+\left(\sum_{R_{i} \notin \mathcal{D}} L_{s r_{i}}\right)\right\}\right)} \\
&\left.\doteq \rho^{(2 r-1)\left(L_{s d}+\min \right.}\left\{\left(\min _{i \in 1, \ldots, K} L_{r_{i} d}\right),\left(\sum_{i=1}^{K} L_{s r_{i}}\right)\right\}\right),
\end{aligned}
$$

where the last line follows from the fact that $\min _{\boldsymbol{R}_{i} \in \mathcal{D}} L_{r_{i} d} \geq \min _{i \in 1, \ldots, K} L_{r_{i} d}$ for any decoding set $\mathcal{D}$. A detailed explanation for the last step in the above derivation follows. Denote

$$
Z(\mathcal{D}) \triangleq \min _{\boldsymbol{R}_{i} \in \mathcal{D}} L_{r_{i} d}+\sum_{\mathbf{R}_{i} \notin \mathcal{D}} L_{s r_{i}} .
$$

Let $\mathcal{D}_{n}$ be a set with $n$ decoding relays so that $\left|\mathcal{D}_{n}\right|=n$. Then, $Z\left(\mathcal{D}_{K}\right)=\min _{i \in 1, \ldots, K} L_{r_{i} d}$ when all the relays are in the decoding set and $Z\left(\mathcal{D}_{0}\right)=\sum_{i \in 1, \ldots, K} L_{s r_{i}}$ when no relay is in the decoding set. For $1 \leq n<K$,

$$
\begin{aligned}
Z\left(\mathcal{D}_{n}\right) & =\min _{\mathbf{R}_{i} \in \mathcal{D}_{n}} L_{r_{i} d}+\sum_{\mathbf{R}_{i} \notin \mathcal{D}_{n}} L_{s r_{i}} \\
& \geq \min _{\mathbf{R}_{i} \in \mathcal{D}_{K}} L_{r_{i} d}+\sum_{\mathbf{R}_{i} \notin \mathcal{D}_{n}} L_{s r_{i}} \\
& \geq \min _{\mathbf{R}_{i} \in \mathcal{D}_{K}} L_{r_{i} d}=Z\left(\mathcal{D}_{K}\right) .
\end{aligned}
$$


Thus, the minimum of $Z(\mathcal{D})$ over all possible decoding sets happens either when $|\mathcal{D}|=K$ or $|\mathcal{D}|=0$. We can write

$$
\min _{\mathcal{D}} Z(\mathcal{D})=\min \left(Z\left(\mathcal{D}_{K}\right), Z\left(\mathcal{D}_{0}\right)\right)=\min \left(\min _{i \in 1, \ldots, K} L_{r_{i} d}, \sum_{i \in 1, \ldots, K} L_{s r_{i}}\right) .
$$

Comparing (29) with (23), we find that the DMT offered by the random selection method is dominated by the best relay selection method. The random selection method cannot always fully exploit the spatial diversity due to the presence of the min in (29) which results in a diversity bottleneck, though we will consider some cases in Section 3.4 where random selection can exploit full spatial diversity.

\subsection{All-decoding-relay DMT}

Next, we analyze the outage of a scheme where all relays in the decoding set participate. Since all decoding relays participate in the forwarding, no overhead, no feedback, and no CSI is needed to perform selection. We assume perfect symbol synchronization now and will comment on this later.

As the decoding relays participate in the second phase of transmission and employ repetition coding, the effective channel from the relays to the destination becomes

$$
\boldsymbol{h}_{r d}=\sum_{\mathbf{R}_{i} \in \mathcal{D}} \boldsymbol{h}_{r_{i} d}
$$

For a fair comparison, we assume each relay transmits at the power of $2 P /|\mathcal{D}|$ where 2 is due to half-duplex relaying. We can write the conditional mutual information between the source and the destination through the decoding set as

$$
I_{\text {all }}=\frac{1}{2} \log \left(1+2 \rho\left(\frac{\left\|\boldsymbol{h}_{r d}\right\|^{2}}{|\mathcal{D}|}+\left\|\boldsymbol{h}_{s d}\right\|^{2}\right)\right) .
$$

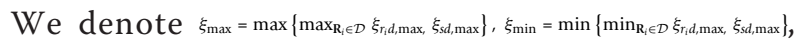
and we can bound $I_{\text {all }}$ as

$$
\frac{1}{2} \log \left(1+2 \rho \xi_{\min }^{2}\left(\frac{\left\|\delta_{r a n}\right\|^{2}}{|\mathcal{D}|}+\left\|\delta_{\delta a l}\right\|^{2}\right)\right) \leq I_{a l l} \leq \frac{1}{2} \log \left(1+2 \rho \xi_{\min }^{2}\left(\frac{\left\|\delta_{r}\right\| \|^{2}}{|\mathcal{D}|}+\left\|\delta_{\delta a d}\right\|^{2}\right)\right),
$$

where $\boldsymbol{\delta}_{r d}=\sum_{\mathbf{R}_{i} \in \mathcal{D}} \boldsymbol{\delta}_{r_{i} d}$ with length $L_{r d} \triangleq \max _{\mathbf{R}_{i} \in \mathcal{D}} L_{r_{i} d}$. Denote the covariance matrix of $\boldsymbol{\delta}_{r d}$ as $C \in \mathbb{R}^{L_{r d} \times L_{r d} \text {. We }}$ note that $C$ is a diagonal matrix with the largest element $|\mathcal{D}|$ and the smallest element greater than or equal to 1 . Define

$$
\overline{\boldsymbol{\delta}}_{r d} \triangleq C^{-1 / 2} \boldsymbol{\delta}_{r d} .
$$

Each element of $\overline{\boldsymbol{\delta}}_{r d}$ is then Gaussian distributed with variance $1,\left\|\overline{\boldsymbol{\delta}}_{r d}\right\|^{2}$ is chi-square distributed with $L_{r d}$ degrees of freedom, and

$$
\left\|\overline{\boldsymbol{\delta}}_{r d}\right\|^{2} \leq\left\|\boldsymbol{\delta}_{r d}\right\|^{2} \leq|\mathcal{D}|\left\|\overline{\boldsymbol{\delta}}_{r d}\right\|^{2} .
$$

Let $Y \triangleq\left\|\delta_{s d}\right\|^{2}$ and $f_{Y}(y)$ be the pdf of $Y$ which is chisquare distributed with $L_{s d}$ degrees of freedom. We develop the conditional outage probability for the alldecoding-relay method as

$$
\begin{aligned}
& \operatorname{Pr}\left[I_{\text {all }}<R \mid \mathcal{D}\right]=\operatorname{Pr}\left[\frac{\left\|\boldsymbol{h}_{r d}\right\|^{2}+Y<b}{|\mathcal{D}|}\right] \\
& \doteq \operatorname{Pr}\left[\frac{\left\|\boldsymbol{\delta}_{r d}\right\|^{2}}{|\mathcal{D}|}+Y<b\right] \\
& \doteq \operatorname{Pr}\left[\left\|\overline{\boldsymbol{\delta}}_{r d}\right\|^{2}+Y<b\right] \\
& \doteq b^{L_{s d}+L_{r d}},
\end{aligned}
$$

where (34) follows by applying (32), (35) follows by applying (33), and (36) follows as $\left\|\overline{\boldsymbol{\delta}}_{r d}\right\|^{2}+Y$ is chisquare distributed with $L_{s d}+L_{r d}$ degrees of freedom. From (36), we see that within the decoding set, dividing power among transmit antennas without phase alignment does not offer spatial diversity and only offers frequency diversity where the diversity order equals the longest delay length.

Substituting (13) and (36) into (12), the outage probability of the all-decoding-relay method is

$$
\begin{aligned}
& \operatorname{Pr}\left[I_{\text {all }}<R\right]=\sum_{\mathcal{D}} \operatorname{Pr}\left[I_{\text {all }}<R \mid \mathcal{D}\right] \operatorname{Pr}[\mathcal{D}] \\
& \doteq \sum_{\mathcal{D}} b^{L_{s d}+\max _{\mathbf{R}_{i} \in \mathcal{D}} L_{r_{i}}+\sum_{\mathbf{R}_{i} \notin \mathcal{D}} L_{s_{i}}} \\
& \doteq \rho^{(2 r-1)\left(L_{s d}+\min _{\mathcal{D}}\left\{\left(\max _{\mathbf{R}_{i} \in \mathcal{D}} L_{r_{i}}\right)+\left(\sum_{\mathbf{R}_{i} \notin \mathcal{D}} L_{s_{i}}\right)\right\}\right)} \text {. }
\end{aligned}
$$

While we assume perfect symbol synchronization, we note that imperfect symbol synchronization has the effect of artificially increasing the channel lengths by adding zeros (or delays) to the front of the impulse responses. The use of intentional asynchronization to induce delay diversity was studied in [27] for the case of flat fading channels. A similar approach could be used in ISI channels; by artificially adding zeros to the front of each component relay-to-destination channel, the effective sum channel from all relays to the destination can be made to have $L_{r d}=\sum_{\mathbf{R}_{i} \in \mathcal{D}} L_{r_{i} d}$ independent paths so that the all-decoding-relay scheme can attain performance equal to the best relay selection if the symbol-level asynchronization is chosen appropriately.

\subsection{Summary}

Collecting the expressions in (24), (29), and (37), we arrive at the DMT expressions for each scheme shown 
in Table 1. By comparing the original outage expressions, it is apparent that

$$
d_{\text {best }}(r) \geq d_{\text {all }}(r) \geq d_{\text {random }}(r) .
$$

Comparing each of these expressions with the DMT upper bound in (9), we see that the best relay selection method is the only one which can always achieve the DMT bound. Table 1 also includes the special case when all source-to-relay channels have identical length $L_{s r}$, and all relay-to-destination channels have identical length $L_{r d}$. We note that our theoretical diversity expressions agree with results reported in elsewhere in the literature. For example, in the special case of flat-fading, our results coincide with those of $[10,11]$ which showed that the best relay selection protocol can achieve diversity equal to $K+1$. Another example is that in [15], with a single relay $K=1$, a system employing STBC can achieve diversity equal to the expression we found for all the three relaying schemes. Additionally, the diversity achieved when using multiple orthogonal relay subchannels in an OFDM system with precoding [20] is identical to the one achieved here by the best relay selection scheme.

It is interesting to note that even random relay selection can achieve the same diversity as best relay selection in some cases. For example, looking at the last column of Table 1, we see that all schemes have an equivalent DMT when $L_{r d}>K L_{s r}$. This situation could arise when there is significant scattering and dispersion in the relay-to-destination channel (due to a high density of large buildings, for example) when compared with the source-to-relay channel (which may have a lower density of reflecting structures and terrain). Thus, when the relay-to-destination channel is sufficiently rich, the lower overhead of random relay selection is attractive. This is different from the situation in flat fading channels, since with $L_{s r}=L_{r d}=1$, best relay selection is the only scheme which can exploit spatial diversity.

The outage probability and DMT bounds derived here are based on the MFB. As the MFB effectively ignores the intersymbol interference, these results provide an optimistic bound on the attainable outage probability and DMT. We now consider a transceiver design for attaining the bound for best relay selection.

\section{Optimal-DMT-achieving transceiver}

In the previous section, we proved that best relay selection can achieve the optimal DMT, the DMT upper bound derived in Section 2. We now propose a specific transmission and reception scheme for best relay selection and we will prove that it can asymptotically achieve the optimal DMT.

\subsection{Transceiver description}

In the proposed scheme, the source sends $N$ QAM-symbols, denoted as $\boldsymbol{x}$, which are drawn from a constellation of $Q=\rho^{2 r^{\prime}}$ points where [28, Equation (2)]

$$
r^{\prime}=\frac{r}{1-\frac{M_{\max ^{-1}}}{N+M_{\max ^{-1}}}}
$$

and

$$
M_{\max } \geq \max _{i \in 1, \ldots, K}\left\{M_{s r_{i}}, M_{r_{i} d}, M_{s d}\right\} .
$$

After transmission of $N$ symbols, a guard interval of length $M_{\max }-1$ zeros follows. The choice of $Q$ or $r^{\prime}$ here is to make sure the total transmission rate is still $R=r \log \rho$ with the guard interval. $M_{\max }$ is essentially an upper bound on the length of all channels in the system. In practice, it is unrealistic for the source node to have knowledge of the lengths of all channels in the system. The system designer needs only choose the parameter $M_{\max }$ to be greater than or equal to the largest channel length expected in the transmission environment. The insertion of guard time eliminates the possibility of interblock interference, but intersymbol interference is still present. Due to the insertion of guard time between alternating phases of source/relay transmission, we see from (38) that the system incurs a rate penalty that can be made arbitrarily small by increasing the block length $N$.

We assume channel state information at the receiver (CSIR) is perfect, but that no channel state information at the transmitter (CSIT) is needed. We also assume perfect frame synchronization though in practice the system can accommodate modest symbol-level synchronization errors since they can be lumped into the FIR channel model. Each relay and the destination uses a ZF equalizer prior to detection to compensate for the intersymbol interference.

In the first phase, the received signal at each relay is

$$
y_{r_{i}}=H_{s r_{i}} x+w_{s r_{i}},
$$

where the $\boldsymbol{H}_{s r_{i}} \in \mathbb{C}^{\left(M_{\max }+N-1\right) \times N}$ are the Toplitz channel convolution matrices corresponding to $\boldsymbol{h}_{s r_{i}}$, i.e. $\left[\boldsymbol{H}_{s r_{i}}\right]_{j, k}=h_{s r_{i}}[j-k]$. Since $\left\|\boldsymbol{h}_{s r_{i}}\right\| \neq 0$ with probability 1 , and the minimum eigenvalue of $\boldsymbol{H}_{s r_{i}}^{H} \boldsymbol{H}_{s r_{i}}$ is greater than zero due to [28, Lemma IV.1], $\boldsymbol{H}_{s r_{i}}^{H} \boldsymbol{H}_{s r_{i}}$ is invertible and the $\mathrm{ZF}$ equalizer coefficients used at the ith relay are

$$
\boldsymbol{G}_{r_{i}}=\left(\boldsymbol{H}_{s r_{i}}^{H} \boldsymbol{H}_{s r_{i}}\right)^{-1} \boldsymbol{H}_{s r_{i}}^{H} .
$$

The filtered estimate of $\boldsymbol{x}$ at each relay is

$$
\begin{aligned}
\tilde{y}_{r_{i}} & =\boldsymbol{G}_{r_{i}} \boldsymbol{y}_{r_{i}} \\
& =x+\left(\boldsymbol{H}_{s r_{i}}^{H} \boldsymbol{H}_{s r_{i}}\right)^{-1} \boldsymbol{H}_{s r_{i}}^{H} \boldsymbol{w}_{s r_{i}} .
\end{aligned}
$$


A given relay is declared to have successfully decoded the message only when each symbol in the block is decoded correctly. The best relay selection scheme described in the previous section is employed, which selects the relay in the decoding set with the largest sumsquared relay-to-destination channel. After the completion of relay selection, in the second phase, the selected relay forwards the length $N$ decoded message to the destination and another guard interval of length $M_{\max }-1$ follows the relayed signal. This process continues and the source sends another block of $N$ symbols. Let the selected relay index be $m$ and denote its relay-destination channel coefficients as $\boldsymbol{h}_{r_{m} d}$ so that

$$
\left\|\boldsymbol{h}_{r_{m} d}\right\|^{2} \triangleq \max _{\mathbf{R}_{i} \in \mathcal{D}}\left\|\boldsymbol{h}_{r_{i} d}\right\|^{2} .
$$

Let $\boldsymbol{H}_{s d} \in \mathbb{C}^{\left(M_{\max }+N-1\right) \times N}, \boldsymbol{H}_{r_{m} d} \in \mathbb{C}^{\left(M_{\max }+N-1\right) \times N}$ be the Toplitz channel convolution matrices corresponding to $\boldsymbol{h}_{s d}$ and $\boldsymbol{h}_{r_{m} d}$, respectively. Define

$$
\boldsymbol{H}_{\mathrm{eff}}=\left[\begin{array}{c}
\boldsymbol{H}_{s d} \\
\boldsymbol{H}_{r_{m} d}
\end{array}\right], \quad \boldsymbol{w}_{\mathrm{eff}}=\left[\begin{array}{c}
\boldsymbol{w}_{s d} \\
\boldsymbol{w}_{r_{m}} d
\end{array}\right] .
$$

Then, the received signal to be equalized at the destination is then given by

$$
y=H_{\text {eff }} x+w_{\text {eff. }}
$$

We note that this model includes the guard intervals inserted between the two transmission phases as can be seen by the dimensions of $\boldsymbol{H}_{s r_{i}}, \boldsymbol{H}_{s d}$, and $\boldsymbol{H}_{r_{m} d}$. We note

$$
\boldsymbol{H}_{\text {eff }}^{H} \boldsymbol{H}_{\mathrm{eff}}=\boldsymbol{H}_{s d}^{H} \boldsymbol{H}_{s d}+\boldsymbol{H}_{r_{m} d}^{H} \boldsymbol{H}_{r_{m} d} .
$$

Denote the minimum eigenvalue of $\boldsymbol{H}_{r_{m} d}^{H} \boldsymbol{H}_{r_{m} d}$ as $\lambda_{r_{m} d, \text { min }}$, the minimum eigenvalue of $\boldsymbol{H}_{s d}^{H} \boldsymbol{H}_{s d}$ as $\lambda_{s d, \mathrm{~min}}$, and the minimum eigenvalue of $\boldsymbol{H}_{\text {eff }}^{H} \boldsymbol{H}_{\text {eff }}$ as $\lambda_{\text {eff,min }}$. From (41) and the fact that these three matrices are Hermitian, Weyl's Inequality [29, Theorem 4.3.1] gives

$$
\lambda_{\text {eff,min }} \geq \lambda_{s d, \min }+\lambda_{r_{m} d, \min } .
$$

Since $\lambda_{s d, \text { min }}>0$ and $\lambda_{r_{m} d, \text { min }}>0$ again due to [28, Lemma IV.1], we have $\lambda_{\text {eff,min }}>0$ and thus $\boldsymbol{H}_{\text {eff }}^{H} \boldsymbol{H}_{\text {eff }}$ is invertible. The destination processes the received signal with a ZF equalizer

$$
\boldsymbol{G}=\left(\boldsymbol{H}_{\text {eff }}^{H} \boldsymbol{H}_{\mathrm{eff}}\right)^{-1} \boldsymbol{H}_{\text {eff }}^{H} .
$$

The filtered estimate of $\boldsymbol{x}$ at the destination is then

$$
\begin{aligned}
\tilde{y} & =G y \\
& =x+\left(H_{\text {eff }}^{H} H_{\text {eff }}\right)^{-1} H_{\text {eff }}^{H} \boldsymbol{w}_{\text {eff. }} .
\end{aligned}
$$

The filtered noise $z=\left(\boldsymbol{H}_{\text {eff }}^{H} \boldsymbol{H}_{\text {eff }}\right)^{-1} \boldsymbol{H}_{\text {eff }}^{H} \boldsymbol{w}_{\text {eff }}$ has total variance

$$
\begin{aligned}
E\left[\|z\|^{2}\right] & =E\left[z^{H} z\right] \\
& =\operatorname{tr}\left[\left(\boldsymbol{H}_{\text {eff }}^{H} \boldsymbol{H}_{\text {eff }}\right)^{-1}\right] N_{0} .
\end{aligned}
$$

\subsection{Outage analysis}

We first analyze the probability of decoding set of this scheme. Define the error probability at the $i$ th relay after ZF equalization as $P_{e, i}$ and denote the minimum eigenvalue of $\boldsymbol{H}_{s r_{i}}^{H} \boldsymbol{H}_{s r_{i}}$ as $\lambda_{s r_{i}}$,min. Following the steps in Theorem III.6 of [28], we have

$$
\begin{aligned}
P_{e, i} & \doteq \operatorname{Pr}\left(\left\|\boldsymbol{h}_{s r_{i}}\right\|^{2}<N \bar{\lambda}_{s r_{i}}^{-1} \rho^{2 r^{\prime}-1}\right) \\
& \leq \operatorname{Pr}\left(\xi_{s r_{i}, \min }^{2}\left\|\boldsymbol{\delta}_{s r_{i}}\right\|^{2}<N \bar{\lambda}_{s r_{i}}^{-1} \rho^{2 r^{\prime}-1}\right) \\
& \doteq \rho^{-L_{s r_{i}}\left(1-2 r^{\prime}\right)},
\end{aligned}
$$

where $\xi_{s r_{i}, \min }$ is the smallest singular value of $\boldsymbol{\Gamma}_{s r_{i}}$, and

$$
\bar{\lambda}_{s r_{i}}=\inf _{h_{s r_{i}} \in \mathbb{C}^{M r_{i}}} \lambda_{s r_{i}, \min }\left(\overline{\boldsymbol{H}}_{s r_{i}}^{H} \overline{\boldsymbol{H}}_{s r_{i}}\right) .
$$

Following the steps in Theorem VII.7 of [28], we have

$$
\begin{aligned}
P_{e, i} & \geq P_{\text {out }, \mathrm{i}} \doteq \operatorname{Pr}\left(\left\|\boldsymbol{h}_{s r_{i}}\right\|^{2}<\rho^{2 r^{\prime}-1}\right) \\
& \geq \operatorname{Pr}\left(\xi_{s r_{i}, \max }^{2}\left\|\boldsymbol{\delta}_{s r_{i}}\right\|^{2}<\rho^{2 r^{\prime}-1}\right) \\
& \doteq \rho^{-L_{s r_{i}}\left(1-2 r^{\prime}\right)},
\end{aligned}
$$

where $\xi_{s r_{i}, \max }$ is the largest singular value of $\boldsymbol{\Gamma}_{s r_{i}}$. Thus, we can conclude

$$
P_{e, i} \doteq \rho^{-L_{s r_{i}}\left(1-2 r^{\prime}\right)} .
$$

As a relay is in the decoding set only when all $N$ symbols are decoded correctly

$$
\operatorname{Pr}\left[\mathbf{R}_{i} \in \mathcal{D}\right]=\left(1-P_{e, i}\right)^{N} .
$$

Thus, the probability of the decoding set is

$$
\begin{aligned}
\operatorname{Pr}[\mathcal{D}] & =\prod_{\mathbf{R}_{i} \notin \mathcal{D}}\left(1-\left(1-P_{e, i}\right)^{N}\right) \prod_{\mathbf{R}_{i} \in \mathcal{D}}\left(1-P_{e, i}\right)^{N} \\
& \doteq \rho^{-\left(1-2 r^{\prime}\right) \sum_{\mathbf{R}_{i} \notin \mathcal{D}} L_{s_{i^{\prime}}}}
\end{aligned}
$$

where asymptotic equality in (45) follows from the binomial theorem. We next analyze the error probability at the destination conditioned on the decoding set. Denote $\lambda_{\text {eff, } k}$ as the $k$ th eigenvalue for $\boldsymbol{H}_{\text {eff }}^{H} \boldsymbol{H}_{\text {eff }}$ with $k \in\{0,1, \ldots, N-1\}$. 
Assume we estimate each symbol in the block separately then the effective $\rho$ for decoding the $k$ th symbol is

$$
\begin{aligned}
\rho_{\text {eff }}(k) & =\frac{P}{W E\left[\left|z_{k}\right|^{2}\right]} \\
& \geq \frac{P}{W E\left[\left\|z_{k}\right\|^{2}\right]} \\
& =\frac{\rho}{\operatorname{tr}\left[\left(\boldsymbol{H}_{\text {eff }}^{H} H_{\text {eff }}\right)^{-1}\right]} \\
& =\frac{\rho}{\sum_{k=0}^{N-1} \lambda_{\text {eff, },}^{-1}} \\
& \geq \frac{\rho}{N \lambda_{\text {eff,min }}^{-1}} \\
& \geq \frac{1}{N} \rho\left(\lambda_{s d, \min }+\lambda_{r_{m} d, \min }\right) \\
& \geq \frac{1}{N} \rho\left(\bar{\lambda}_{s d, \min }\left\|\boldsymbol{h}_{s d}\right\|^{2}+\bar{\lambda}_{r_{m} d, \min }\left\|\boldsymbol{h}_{r_{m} d}\right\|^{2}\right) \\
& \geq \frac{1}{N} \rho \bar{\lambda}\left(\left\|\boldsymbol{h}_{s d}\right\|^{2}+\left\|\boldsymbol{h}_{r_{m} d}\right\|^{2}\right),
\end{aligned}
$$

where $\bar{\lambda}=\min \left(\bar{\lambda}_{s d}, \bar{\lambda}_{r_{m} d}\right)$ with

$$
\begin{aligned}
& \bar{\lambda}_{s d}=\inf _{h_{s d} \in \mathbb{C}^{M_{s d}}} \lambda_{s d, \min }\left(\overline{\boldsymbol{H}}_{s d}^{H} \overline{\boldsymbol{H}}_{s d}\right), \\
& \lambda_{r_{m} d}=\inf _{h_{r_{m} d} \in \mathbb{C}^{M_{r m} d}} \lambda_{r_{m} d, \min }\left(\overline{\boldsymbol{H}}_{r_{m} d}^{H} \overline{\boldsymbol{H}}_{r_{m} d}\right),
\end{aligned}
$$

and

$$
\overline{\boldsymbol{H}}_{s d} \triangleq \frac{\boldsymbol{H}_{s d}}{\left\|\boldsymbol{h}_{s d}\right\|^{\prime}}, \quad \overline{\boldsymbol{H}}_{r_{m} d} \triangleq \frac{\boldsymbol{H}_{r_{m} d}}{\left\|\boldsymbol{h}_{r_{m} d}\right\|} .
$$

Using a proof identical to [28, Lemma IV.1], it can be shown that $\bar{\lambda}_{s d}>0$ and $\bar{\lambda}_{r_{m} d}>0$, therefore $\bar{\lambda}>0$.

The error probability at the destination conditioned on $\mathcal{D}$ [28, Lemma VII.6] is

$$
\begin{aligned}
& P_{e \mid \mathcal{D}} \doteq \operatorname{Pr}\left[\rho_{\text {eff }}(k)<\rho^{2 r^{\prime}} \mid \mathcal{D}\right] \\
& \leq \operatorname{Pr}\left[\left(\left\|\boldsymbol{h}_{s d}\right\|^{2}+\left\|\boldsymbol{h}_{r_{m} d}\right\|^{2}\right) \frac{1}{N} \rho \bar{\lambda}<\rho^{2 r^{\prime}}\right] \\
&=\operatorname{Pr}\left[\left(\left\|\boldsymbol{h}_{s d}\right\|^{2}+\max _{\mathbf{R}_{i} \in \mathcal{D}}\left\|\boldsymbol{h}_{r_{i} d}\right\|^{2}\right)<\frac{N}{\lambda} \rho^{2 r^{\prime}-1}\right] \\
& \doteq \operatorname{Pr}\left[\left(\left\|\boldsymbol{\delta}_{s d}\right\|^{2}+\max _{\mathbf{R}_{i} \in \mathcal{D}}\left\|\boldsymbol{\delta}_{r_{i} d}\right\|^{2}\right)<\frac{N}{\lambda} \rho^{2 r^{\prime}-1}\right] \\
& \doteq \rho^{\left(2 r^{\prime}-1\right)\left(L_{s d}+\sum_{\mathbf{R}_{i} \in \mathcal{D}} L_{r_{i} d}\right),}
\end{aligned}
$$

where (46) follows by applying (16), and the last step comes from steps identical to (17)-(22). Combining (45) and (47) by the total probability theorem, we conclude that the proposed transmission scheme and equalization method have the following upper bound on the error probability:

$$
\begin{aligned}
P_{e} & \doteq \sum_{\mathcal{D}} P_{e \mid \mathcal{D}} \operatorname{Pr}[\mathcal{D}] \\
& \doteq \sum_{\mathcal{D}} \rho^{\left(2 r^{\prime}-1\right)\left(L_{s d}+\sum_{\mathrm{R}_{i} \in \mathcal{D}} L_{r_{i} d}+\sum_{\mathrm{R}_{i} \notin \mathcal{D}} L_{s r_{i}}\right)} \\
& \doteq \rho^{\left(2 r^{\prime}-1\right)\left(L_{s d}+\sum_{i=1}^{K} \min \left(L_{r_{i} d} L_{s_{i}}\right)\right)} .
\end{aligned}
$$

Combining [28, Lemma III.1] and the result in (24), we also have

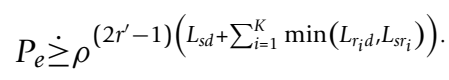

Thus, we can conclude that

$$
P_{e} \doteq \rho^{\left(2 r^{\prime}-1\right)\left(L_{s i}+\sum_{i=1}^{K} \min \left(L_{r_{i}}, L_{s s_{i}}\right)\right)} \text {. }
$$

which shows that the proposed scheme can asymptotically achieve the DMT for best relay selection.

We also point out that since minimum mean squarederror (MMSE) and decision feedback equalizer (DFE) performance dominates ZF equalizers [30], MMSE equalizers and DFEs should attain the same DMT curve. In practice, a system designer may prefer a MMSE or DFE equalizer for their improved BER performance.

\section{Numerical results}

This section presents numerical examples of the performance of the proposed relay selection methods developed in Sections 3 and 4. In evaluating performance over finite SNRs, the diversity measured as the negative slope of each outage curve often does not coincide exactly with the predicted maximal diversity [31,32]; the predicted diversity assumes that the SNR grows arbitrarily large to permit the analysis to be mathematically tractable. We now compare the performance of the three selection schemes in a variety of scenarios at finite SNR, and show that the schemes follow the general trends predicted by the DMT results.

To illustrate the attainable frequency and spatial diversity, we consider 10 scenarios shown in Table 2 where the maximum diversity order is computed from (24), (30), and (37). The first five scenarios-which use a single relay and therefore are unaffected by the choice of selection strategy-are included to illustrate the relative improvement in spatial diversity by adding additional relays.

The outage probability for the best relay selection, random relay selection, and all-decoding-relay methods are plotted in Figures 3, 4, and 5, respectively, where the rate $R=2 \mathrm{bits} / \mathrm{s} / \mathrm{Hz}$ and each $\boldsymbol{\Gamma}_{j k}$ is a square identity matrix, i.e. each fading tap of $\boldsymbol{h}_{\boldsymbol{j} k}$ is i.i.d. Rayleigh fading with variance 1 . For the best relay selection performance 
Table 2 Simulation scenarios

\begin{tabular}{cccccccc}
\hline Scenario & $\mathbf{K}$ & $\mathbf{L}_{\mathbf{s d}}$ & $\mathbf{L}_{\mathbf{s r}}$ & $\mathbf{L}_{\mathbf{r d}}$ & $\mathbf{d}_{\mathbf{m a x}, \text { best }}$ & $\mathbf{d}_{\mathbf{m a x}, \text { random }}$ & $\mathbf{d}_{\mathbf{m a x}, \text { all }}$ \\
\hline 1 & 1 & 2 & 2 & 2 & 4 & 4 & 4 \\
2 & 1 & 2 & 2 & 3 & 4 & 4 & 4 \\
3 & 1 & 2 & 3 & 2 & 4 & 4 & 4 \\
4 & 1 & 2 & 4 & 4 & 6 & 6 & 6 \\
5 & 1 & 4 & 2 & 2 & 6 & 6 & 6 \\
6 & 2 & 2 & 2,2 & 2,2 & 6 & 4 & 4 \\
7 & 2 & 2 & 2,2 & 3,3 & 6 & 5 & 5 \\
8 & 2 & 2 & 3,3 & 2,2 & 6 & 4 & 4 \\
9 & 2 & 2 & 4,4 & 4,4 & 10 & 6 & 6 \\
10 & 2 & 4 & 2,2 & 2,2 & 8 & 6 & 6 \\
\hline
\end{tabular}

shown in Figure 3, the outage curves for Scenarios 1 through 3 have roughly the same slope which agrees with the trend predicted by Table 2 . Scenario 4 has a higher diversity order since the minimum length of the source-to-relay and relay-to-destination channels increases, and Scenario 5 has an increased diversity order due to the increased length of the source-to-destination channel. Similar behavior is observed for Scenarios 6 through 10, which have correspondingly larger diversity orders than Scenarios 1 through 5 because of the spatial diversity offered by the additional relay. We also notice that increasing the frequency diversity in the source-to-relay channel results in a more pronounced coding gain than increasing the frequency diversity in the relay-to-destination channel, as Scenario 3 has a larger coding gain than Scenario 2 in Figure 3. As shown by Figures 4 and 5, the random relay selection and alldecoding-relay methods have nearly the same outage performance as each other for the considered scenarios; this is another trend predicted by the maximum diversity order in Table 2. Furthermore, both of these schemes have outage performance dominated by the best relay selection method as expected.

We further verify the performance of the transceiver design with different relaying strategies. In the simulation, we use a block length $N=32$ and Gray-mapped QPSK modulation. In Figures 6 and 7, we consider a frequency selective channel with uniform power delay profile, i.e. each tap of each channel is i.i.d. fading with variance $1 / L$ where $L$ is the channel length. On both figures, we have also included the performance of the optimal maximum likelihood sequence estimator (MLSE) equalizer for comparison. As we can see from Figure 6, with only two relays present in the system, the performance advantage of best relay selection over the other two relaying strategies is negligible. This suggests that in systems with a relatively small number of relays, selection strategies that do not require feedback or CSI (such as the random relay selection and all-decoding-relay methods) may be preferred for their simplicity.

When as many as $K=10$ relays are available, as shown in Figure 7, the diversity order of the best relay selection may be significantly larger than the other two methods. In examining the power gain of best relay selection over the other two relaying strategies, we note an interesting trend. When the fading channels contain $L=2$ taps, the power gain of the best relay selection is about $6 \mathrm{~dB}$ at a bit error rate of $10^{-6}$. When $L$ increases to 4 , however, the power gain of best relay selection is only about $2 \mathrm{~dB}$. Thus, when there is already sufficient



Figure 3 Simulated outage probability for the best relay selection method, $R=2 \mathrm{bits} / \mathrm{s} / \mathrm{Hz}$ 


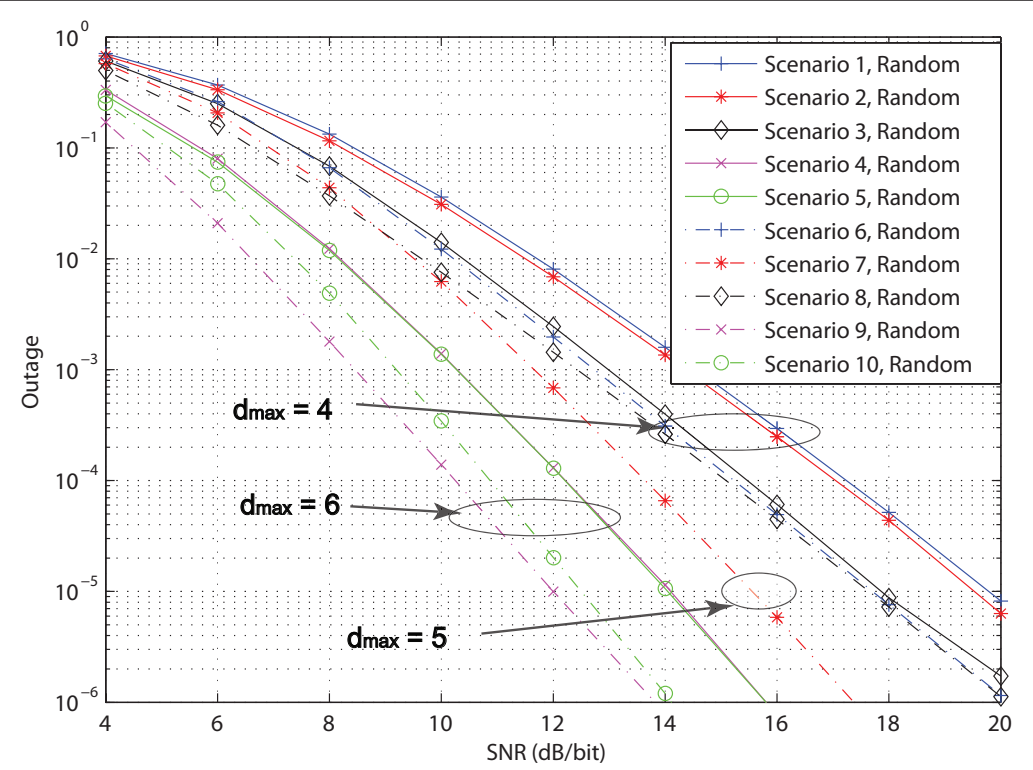

Figure 4 Simulated outage probability for random relay selection method, $R=2 \mathrm{bits} / \mathrm{s} / \mathrm{Hz}$.

frequency diversity in the system, the improvement in using sophisticated selection schemes which better exploit the spatial diversity is not as pronounced. Again, a system designer may favor one of the simpler selection schemes if it is known that the transmission environment has sufficient frequency diversity.

We next compare the BER performance of different relaying strategies under i.i.d. and correlated fading. The i.i.d. fading channel used for comparison has uniform power delay profile, and its performance is shown in Figures 6 and 7 with $L=4$, and is referred to as "no correlation" in Figure 8. To introduce the correlation in the channel taps, we model each channel in the system as a GSM typical rural channel [33] which has $L=4$ underlying independent fading coefficients where each of the four path arrivals have power delay profile given by $[0,-2,-10,-20] \mathrm{dB}$ and corresponding path arrival times $\boldsymbol{\tau}^{T}=[0,0.2,0.4,0.6] \mu \mathrm{s}$. We employ a square root raised cosine pulse shape $p(t)$ at the transmitting end with rolloff factor 0.4 which is truncated to a length of 8 symbol periods. The symbol period is taken to be $T=$ $0.278 \mu$ s which, with QPSK transmission, corresponds to

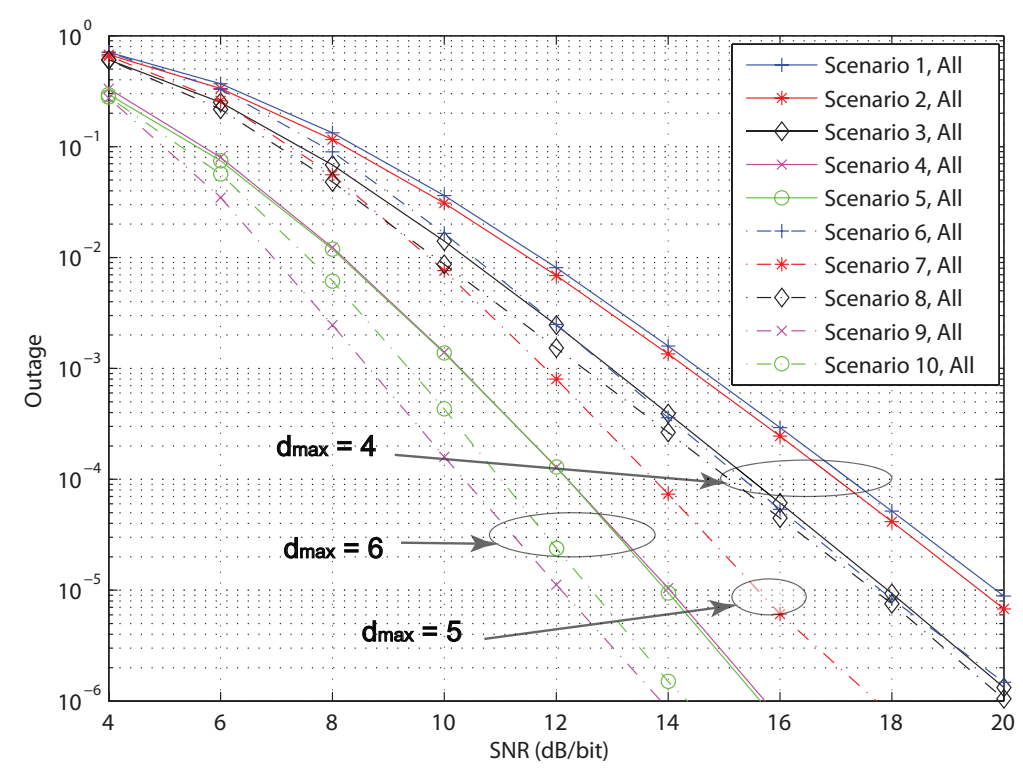

Figure 5 Simulated outage probability for all-decoding-relay method, $R=2 \mathrm{bits} / \mathrm{s} / \mathrm{Hz}$. 


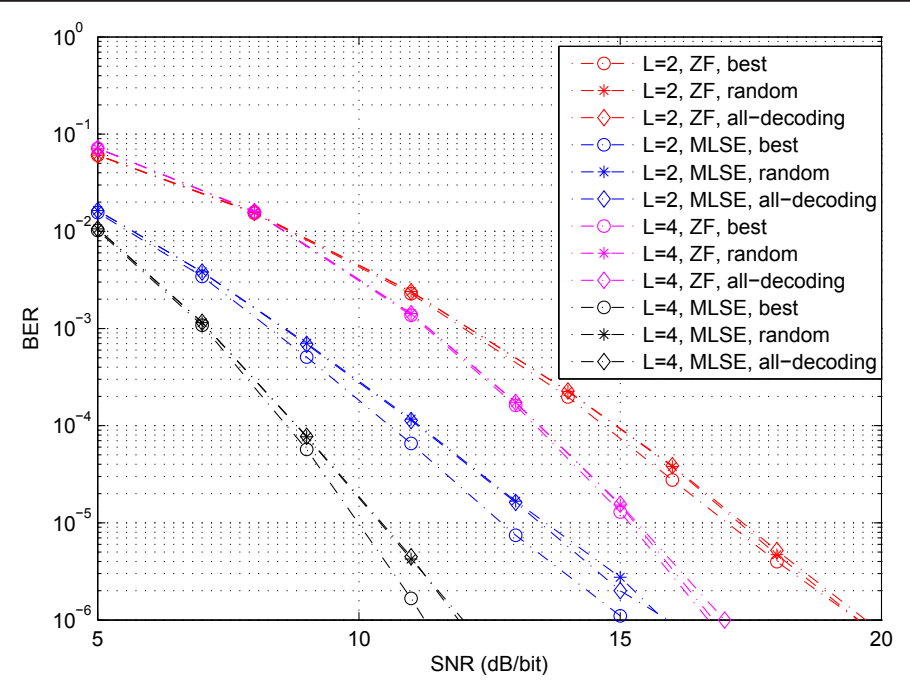

Figure 6 Simulated BER for i.i.d. fading channels, $K=2$.

a data rate of 7.2 Mbps. Together, these parameters determine the tap autocovariance $\boldsymbol{\Gamma}_{j k}$ for each discrete baseband channel since each sampled channel $\boldsymbol{h}$ arises as

$$
h[n]=\left.h(t)\right|_{t=n T}=\left.\sum_{\ell=0}^{L-1} \alpha_{\ell} p\left(t-\tau_{\ell}\right) p(-t)\right|_{t=n T},
$$

where the underlying independent fading coefficients $\alpha \in \mathbb{C}^{4}$ are complex Gaussian with variance given by the GSM typical rural power delay profile. For fair comparison, we normalize the total average power in the underlying independent fading coefficients to 1 . The resulting sampled channel with four independent path arrivals gives rise to a correlated discrete channel with
19 taps. As shown in Figure 8, the simulated performance demonstrates that at finite SNR the receiver is not able to exploit the diversity offered by all four independent paths since the last path which has a power of $-20 \mathrm{~dB}$ contributes very little to the received signal, an effect masked by the high-SNR analysis of the DMT. Nevertheless, the choice of relay selection method still has significant impact on system performance when the number of relays $K$ is relatively large.

\section{Conclusion}

In this article, we have considered the relay selection problem for the orthogonal decode-and-forward system where correlated frequency selective fading is present. We analyzed the outage performance and derived the

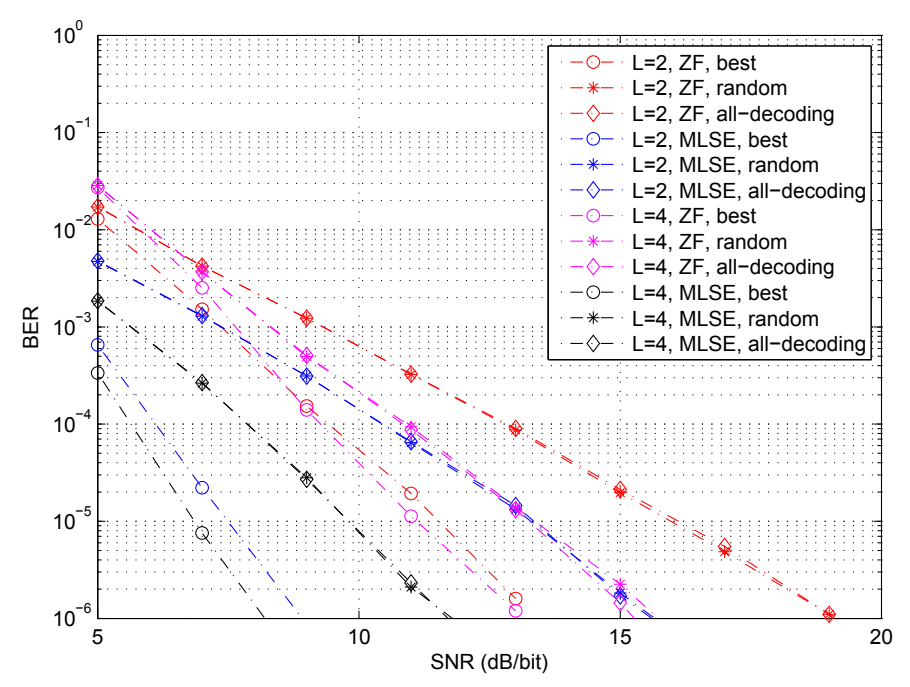

Figure 7 Simulated BER for i.i.d. fading channels, $K=10$. 


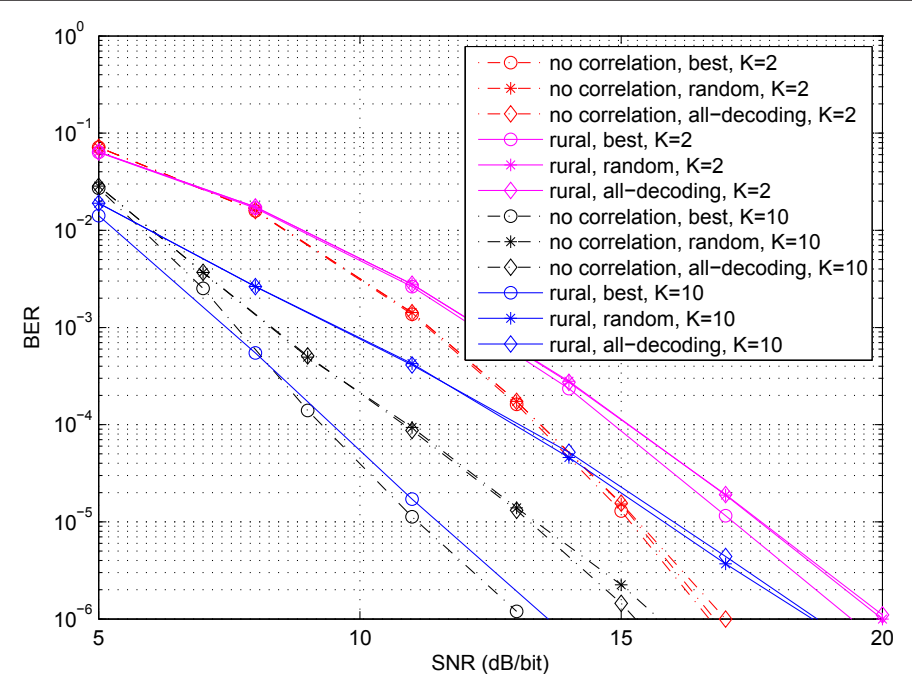

Figure 8 Simulated BER for correlated fading channels.

DMT for three relay methods: best relay selection, random relay selection, and the all-decoding-relay method. Our analysis shows that best relay selection performance dominates the other two schemes with respect to outage. We further proposed a transceiver to realize the DMT offered by best relay selection with minimal complexity; the proposed scheme uses uncoded QAM transmission with guard times and uses ZF equalization at each node. The analysis and simulation results show that the proposed scheme asymptotically achieves the DMT.

While the diversity offered by relay systems in flat fading channels is fairly well understood, the deployment of relay systems in ISI channels requires consideration of a variety of new issues in order to best exploit the available diversity. For example, we presented cases where random relay selection and the all-decoding-relay method can achieve the same diversity as best relay selection, which runs counter to the situation in flat fading channels where best relay selection is always superior. We also found that only when the number of relays in the system is relatively large, the best relay selection offers a significant performance advantage over the other relaying strategies, though this tends to diminish with increased frequency diversity in the system. As the overhead of random relay selection is lower than that of the best relay selection, system designers may favor random relaying depending on the application and transmission environment.

The analytical study presented here focuses on the high-SNR regime and is an important step toward understanding the diversity offered by relay systems in frequency selective fading channels. The relaying strategies presented in this article do not require sophisticated space-time coding, they have relaxed synchronization requirements, and are spectrally efficient; these advantages make the relay selection methods ready for implementation in today's distributed networks. Future study may consider the use of alternate forwarding protocols (such as amplify-and-forward or equalize-and-forward) as well as the overhead tradeoff of the various relay selection methods.

\section{Competing interests}

The authors declare that they have no competing interests.

Received: 6 April 2011 Accepted: 16 November 2011 Published: 16 November 2011

\section{References}

1. A Sendonaris, E Erkip, B Aazhang, User cooperation diversity-part I: system description. IEEE Trans Commun. 51(11), 1927-1938 (2003). doi:10.1109/ TCOMM.2003.818096

2. A Sendonaris, E Erkip, B Aazhang, User cooperation diversity-part II. Implementation aspects and performance analysis. IEEE Trans Commun. 51(11), 1939-1948 (2003). doi:10.1109/TCOMM.2003.819238

3. R Nabar, H Bolcskei, F Kneubuhler, Fading relay channels: performance limits and space-time signal design. IEEE J Sel Areas Commun. 22(6), 1099-1109 (2004). doi:10.1109/JSAC.2004.830922

4. J Laneman, D Tse, G Wornell, Cooperative diversity in wireless networks: efficient protocols and outage behavior. IEEE Trans Inf Theory. 50(12), 3062-3080 (2004). doi:10.1109/TIT.2004.838089

5. A Host-Madsen, J Zhang, Capacity bounds and power allocation for wireless relay channels. IEEE Trans Inf Theory. 51(6), 2020-2040 (2005). doi:10.1109/TIT.2005.847703

6. K Azarian, H El Gamal, P Schniter, On the achievable diversity-multiplexing tradeoff in half-duplex cooperative channels. IEEE Trans Inf Theory. 51(12), 4152-4172 (2005). doi:10.1109/TIT.2005.858920

7. J Laneman, G Wornell, Distributed space-time-coded protocols for exploiting cooperative diversity in wireless networks. IEEE Trans Inf Theory. 49(10), 2415-2425 (2003). doi:10.1109/TIT.2003.817829

8. Y Shang, X-G Xia, Shift-full-rank matrices and applications in space-time trellis codes for relay networks with asynchronous cooperative diversity. IEEE Trans Inf Theory. 52(7), 3153-3167 (2006)

9. A Bletsas, A Khisti, Win M, Opportunistic cooperative diversity with feedback and cheap radios. IEEE Trans Wireless Commun. 7(5), 1823-1827 (2008) 
10. A Bletsas, A Khisti, D Reed, A Lippman, A simple cooperative diversity method based on network path selection. IEEE J Sel Areas Commun. 24(3), 659-672 (2006)

11. E Beres, R Adve, On selection cooperation in distributed networks, in 40th Annual Conference on Information Sciences and Systems. 1056-1061 (March 2006)

12. L Zheng, D Tse, Diversity and multiplexing: a fundamental tradeoff in multiple-antenna channels. IEEE Trans Inf Theory. 49(5), 1073-1096 (2003). doi:10.1109/TIT.2003.810646

13. A Bletsas, H Shin, M Win, Cooperative communications with outage-optimal opportunistic relaying. IEEE Trans Wireless Commun. 6(9), 3450-3460 (2007)

14. D Tse, P Viswanath, Fundamentals of Wireless Communication (Cambridge University Press, Cambridge, 2005)

15. H Mheidat, M Uysal, N Al-Dhahir, Equalization techniques for distributed space-time block codes with amplify-and-forward relaying. IEEE Trans Signal Process. 55(5), 1839-1852 (2007)

16. W Zhang, Y Li, X-G Xia, P Ching, K Ben Letaief, Distributed space-frequency coding for cooperative diversity in broadband wireless ad hoc networks. IEEE Trans Wireless Commun. 7(3), 995-1003 (2008)

17. B Gui, L Cimini, L Dai, OFDM for cooperative networking with limited channel state information. in Military Communications Conference (MILCOM'06) 1-6 (October 2006)

18. L Dai, B Gui, L Cimini, Selective relaying in OFDM multihop cooperative networks. in Wireless Communications and Networking Conference 963-968 (March 2007)

19. Z Wang, G Giannakis, Complex-field coding for OFDM over fading wireless channels. IEEE Trans Inf Theory. 49(3), 707-720 (2003). doi:10.1109/ TIT.2002.808101

20. Y Ding, M Uysal, Amplify-and-forward cooperative OFDM with multiplerelays: performance analysis and relay selection methods. IEEE Trans Wireless Commun. 8(10), 4963-4968 (2009)

21. M Gastpar, M Vetterli, On the capacity of large Gaussian relay networks. IEEE Trans Inf Theory. 51(3), 765-779 (2005). doi:10.1109/TIT.2004.842566

22. A Medles, D Slock, Optimal diversity vs multiplexing tradeoff for frequency selective MIMO channels. in International Symposium on Information Theory (ISIT'05) 1813-1817 (September 2005)

23. S Zhou, G Giannakis, Space-time coding with maximum diversity gains over frequency-selective fading channels. IEEE Signal Process Lett. 8(10), 269-272 (2001). doi:10.1109/97.957268

24. T Cormen, CE Leiserson, DL Rivest, C Stein, Introduction to Algorithms (The MIT press, Cambridge, 2001)

25. S Yang, J Belfiore, Diversity of MIMO multihop relay channels. http://arxiv. org/PS_cache/arxiv/pdf/0708/0708.0386v1.pdf

26. I Gradshteyn, I Ryzhik, A Jeffrey, D Zwillinger, Table of Integrals Series and Products (Academic Press, New York, 2007)

27. S Wei, D Goeckel, M Valenti, Asynchronous cooperative diversity. IEEE Trans Wireless Commun. 5(6), 1547-1557 (2006)

28. L Grokop, D Tse, Diversity-multiplexing tradeoff in ISI channels. IEEE Trans Inf Theory. 55(1), 109-135 (2009)

29. R Horn, C Johnson, Matrix Analysis (Cambridge University Press, Cambridge, 1990)

30. JR Barry, E Lee, D Messerschmitt, Digital Communication (Springer, Netherlands, 2004)

31. R Narasimhan, Finite-SNR diversity-multiplexing tradeoff for correlated Rayleigh and Rician MIMO channels. IEEE Trans Inf Theory. 52(9), 3965-3979 (2006)

32. K Azarian, H El Gamal, The throughput-reliability tradeoff in block-fading MIMO channels. IEEE Trans Inf Theory. 53(2), 488-501 (2007)

33. 3GPP, http://www.3gpp.org/ftp/Specs/html-info/45005.htm (2011). Technical specification group GSM/EDGE radio access network; radio transmission and reception (release 10), 3rd Generation Partnership Project (3GPP), TS 45.005

doi:10.1186/1687-1499-2011-171

Cite this article as: Deng and Klein: Relay selection in cooperative networks with frequency selective fading. EURASIP Journal on Wireless Communications

and Networking 2011 2011:171.

\section{Submit your manuscript to a SpringerOpen ${ }^{\circ}$ journal and benefit from:}

- Convenient online submission

- Rigorous peer review

- Immediate publication on acceptance

- Open access: articles freely available online

- High visibility within the field

- Retaining the copyright to your article

Submit your next manuscript at $\gg$ springeropen.com 